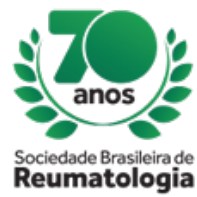

\title{
MAYARO FEVER: AN IMMEDIATE OUTBREAK OF ANOTHER ARBOVIROSIS?
}

Ana Mel Rocha (Centro Universitário de João Pessoa, João Pessoa, PB, Brasil), Ana Caroline Delmondes (Centro Universitário de João Pessoa, João Pessoa, PB, Brasil), Bianca Kelly de França (Centro

Universitário de João Pessoa, João Pessoa, PB, Brasil), Bruno Antunes de Figueiredo (Centro Universitário de João Pessoa, João Pessoa, PB, Brasil), Gabriella Bento de Morais (Centro Universitário de João Pessoa, João Pessoa, PB, Brasil), Laísa Angélica Souto (Centro Universitário de João Pessoa, João Pessoa, PB, Brasil), Simeão Fernandes Cananéa (Centro Universitário de João Pessoa, João Pessoa, PB, Brasil), Pablo Duarte Lima (Centro Universitário de João Pessoa, João Pessoa, PB, Brasil), Teresa Patrícia Acebey (Centro Universitário de João Pessoa, João Pessoa, PB, Brasil)

\section{BACKGROUND}

The Mayaro arbovirus (MAYV) is enzootic of tropical rainforests in central and southern America. It is responsible for clinic characterized by the triad: abrupt fever, prolonged arthralgia and maculopapular rash. The primary vector is Haemagogos janthinomys, with possible participation of mosquitoes of the genus Culex and Aedes in the transmission of the disease. Originally described in Trinidad and Tobago in 1954; In Brazil, in 1955, the first outbreak was in Pará. Recently, new cases have been registered in the Amazon Region and in the Central Plateau of the Brazilian territory.

\section{MATERIALS AND METHODS}

This is a Literary Review study based on articles in the Pubmed, BVS and Scielo databases, carried out between april and june 2019. Inclusion criteria were: publications in the last five years and in English, Spanish and Portuguese. The descriptors used were: mayaro, arthralgia, outbreak. As exclusion criteria: case reports, titles and summaries of low relevance on the subject; being selected 14 articles.

\section{RESULTS}

Recent studies show a clinical picture of Mayaro Fever similar to other arboviruses. Therefore, the differences between clinical and molecular diagnosis have been debated. Symptoms have been described as acute onset fever, arthralgia, maculopapular rash, in addition to headache, myalgia, retro-ocular pain and vomiting; requiring an accurate diagnosis. To do so, the diagnostic methods used are neutralization, ELISA and polymerase chain reaction (PCR) tests. Between 2014 and 2015, 56\% were positive for MAYV in Goiânia / GO. Research indicates that this result is due to the fact that in urban areas, the vector for MAYV would be Aedes aegypti - a mosquito that appears cosmopolitan, serving as a disseminator of diseases for large population groups. Observational studies have shown that in 200 blood samples from patients with acute febrile illness in Sinop / MT, 38 were positive for dengue and six for MAYV. These had received clinical diagnosis of dengue, evidencing the overestimation of this disease and little evidence for other arboviruses.

\section{CONCLUSION}

In the last decade Brazil has been a scenario for the development of arboviruses, and adding to this factor the environment conducive to the development of the vector and the confirmed presence of cases already diagnosed in the Brazilian territory, it is essential to carefully monitor the evolution of the MAYV. Therefore, it is fundamental to understand its pathogenesis and immune response, as well as to put into practice the lessons learned from the other arbovirus outbreaks, in order to minimize the socioeconomic damages. 